University of Nebraska - Lincoln

DigitalCommons@University of Nebraska - Lincoln

May 2002

\title{
The finite-temperature densities of states for half-metallic ferromagnets
}

\author{
Ralph Skomski \\ University of Nebraska-Lincoln, rskomski2@unl.edu \\ Peter A. Dowben \\ University of Nebraska-Lincoln, pdowben@unl.edu
}

Follow this and additional works at: https://digitalcommons.unl.edu/physicsdowben

Part of the Physics Commons

Skomski, Ralph and Dowben, Peter A., "The finite-temperature densities of states for half-metallic ferromagnets" (2002). Peter Dowben Publications. 91.

https://digitalcommons.unl.edu/physicsdowben/91

This Article is brought to you for free and open access by the Research Papers in Physics and Astronomy at DigitalCommons@University of Nebraska - Lincoln. It has been accepted for inclusion in Peter Dowben Publications by an authorized administrator of DigitalCommons@University of Nebraska - Lincoln. 
Europhys. Lett., 58 (4), pp. 544-548 (2002)

\title{
The finite-temperature densities of states for half-metallic ferromagnets
}

\author{
R. Skomski and P. A. Dowben \\ Department of Physics and Astronomy and Center for Materials Research and Analysis, \\ University of Nebraska Lincoln - Lincoln, NE 68588, USA
}

(received 17 October 2001; accepted in final form 27 February 2002)

PACS. 71.20.Lp - Intermetallic compounds.

PACS. 73.20.At - Surface states, band structure, electron density of states.

PACS. 75.25.+z - Spin arrangements in magnetically ordered materials (including neutron and spin-polarized electron studies, synchrotron-source X-ray scattering, etc.).

\begin{abstract}
A unitary spinor transformation is used to show that the spin-down density of states (DOS) of half-metallic ferromagnets becomes nonzero at finite temperatures. In a strict sense, this thermally activated spin mixing means that half-metallic ferromagnetism is limited to zero temperature. Similar effects are created by crystal imperfections, interfaces, and surfaces. The calculations, which imply that the finite-temperature resistivity of an individual spin channel is never infinite, are used to discuss recent experimental work on half-metallic oxides and Heusler-type alloys.
\end{abstract}

Half-metallic ferromagnets have only one spin channel for conduction. Because the resistance of the spin-down channel goes to infinity for $D \downarrow\left(E_{\mathrm{F}}\right)=0$, they are of great importance in spin electronics. Recently, the existence of half-metallic behavior at finite temperatures has been questioned on experimental grounds [1,2], and in spite of several claims of experimental proof of half-metallic character [3-9] there is no compelling experimental proof of half-metallic character for any system. This letter provides a likely explanation of these findings.

Far below $T_{\mathrm{c}}$, the densities of states (DOS) of typical ferromagnets are only weakly modified, because $k_{\mathrm{B}} T$ is much smaller than the Fermi energy and band-structure distortions are small. However, we will see that half-metallic ferromagnets are an exception, with qualitatively new temperature-dependent effects.

To describe the effect of finite temperatures on the band structure, we use the tight-binding Hamiltonian

$$
\mathcal{H}_{\mu \nu i k \sigma \sigma^{\prime}}=\mathcal{T}_{\mu \nu i k} \delta_{\alpha \beta}-V_{\mu} \delta_{\mu \nu} \delta_{i k} \delta_{\alpha \beta}-I_{0} \delta_{\mu \nu} \delta_{i k} \boldsymbol{e}_{i} \cdot \boldsymbol{\sigma}_{\alpha \beta}
$$

Here $\mathcal{T}_{\mu \nu i k}=\left\langle\phi_{\mu}\left(\boldsymbol{r}-\boldsymbol{R}_{i}\right)|\mathcal{T}| \phi_{\nu}\left(\boldsymbol{r}-\boldsymbol{R}_{k}\right)\right\rangle$ is the hopping integral between a $\mu$-type orbital at $\boldsymbol{R}_{i}$ and a $\nu$-type orbital at $\boldsymbol{R}_{k}, V_{\mu}$ is the crystal-field energy for the $\mu$-type orbital, $I_{0}$ is the intra-atomic $d$-electron exchange, and $\boldsymbol{e}_{i}$ is the local magnetization direction. In terms of eq. (1), ferromagnetism results from the $I_{0}$ Stoner term, which reduces and enhances the energies of $\uparrow$ and $\downarrow$ electrons, respectively. In the absence of spin disorder, the spin structure (C) EDP Sciences 
of a ferromagnet is described by the Pauli matrix $\sigma_{z}$ whose $\uparrow$ and $\downarrow$ eigenfunctions are $(1,0)^{T}$ and $(0,1)^{T}$, respectively, and yields an exchange-energy difference of $2 I_{0}$ between spin-up and spin-down electrons. The starting point for describing finite-temperature spin disorder is the standard spin-(1/2) rotation matrix

$$
\hat{U}(\phi, \theta)=\left(\begin{array}{cc}
\cos \left(\frac{\theta}{2}\right) e^{i \theta / 2} & \sin \left(\frac{\theta}{2}\right) e^{-i \phi / 2} \\
-\sin \left(\frac{\theta}{2}\right) e^{i \phi / 2} & \cos \left(\frac{\theta}{2}\right) e^{-i \phi / 2}
\end{array}\right),
$$

which rotates a $\uparrow$ spin by the angles $\theta$ and $\phi[10]$. Note that expressions of the type (1) and (2) apply not only to thermal disorder but also to structural disorder [10-12]; we will exploit this analogy in our discussion of recent experimental work.

The $\boldsymbol{e}_{i} \cdot \boldsymbol{\sigma}_{\alpha \beta}$ exchange term in eq. (1) is diagonalized by the inverse matrix $\hat{U}^{-}=\hat{U}^{+}\left(\phi_{i}, \theta_{i}\right)$. Applying the transformation $\hat{U}_{\mathrm{t}}=\hat{U}^{+}\left(\phi_{1}, \theta_{1}\right) \cdots \hat{U}^{+}\left(\phi_{i} \theta_{i}\right) \hat{U}^{+}\left(\phi_{i+1}, \theta_{i+1}\right) \cdots \hat{U}^{+}\left(\phi_{N}, \theta_{N}\right)$ to eq. (1) yields

$$
\mathcal{H}_{\mu \nu i k \alpha \beta}^{\mathrm{t}}=\mathcal{T}_{\mu \nu i k} \Sigma_{\gamma} U_{\alpha \gamma}\left(\phi_{i}, \theta_{i}\right) U^{+}{ }_{\gamma \beta}\left(\phi_{k}, \theta_{k}\right)-\left(V_{\mu} \delta_{\alpha \beta}+I_{0} \sigma_{z \alpha \beta}\right) \delta_{\mu \nu} \delta_{i k},
$$

where the thermal spin disorder has been mapped onto random hopping integrals.

In the low-temperature regime, dominated by long-wavelength spin waves, neighboring spins remain nearly parallel. Using $\hat{U}\left(\phi_{k}, \theta_{k}\right) \approx \hat{U}\left(\phi_{i}, \theta_{i}\right)$ and exploiting the identity $\hat{U}\left(\phi_{i}, \theta_{i}\right) \hat{U}^{+}\left(\phi_{i}, \theta_{i}\right)=\hat{I}$, we find that $\hat{U}\left(\phi i, \theta_{i}\right) \hat{U}^{+}\left(\phi_{k}, \theta_{k}\right) \approx \hat{I}$. This means that the transformed hopping integrals are very similar to those at zero temperature, and the total wave function obtained via $\hat{U}_{\mathrm{t}}$ is a good approximation. Figure 1a shows typical finite-temperature $\uparrow$ and $\downarrow$ densities of state. The $\uparrow$ DOS exhibits, in general, minor distortions (dark region I), but in the $\downarrow$ gap the DOS changes qualitatively, from zero to nonzero (dark region II). This means that resistance of the $\downarrow$ channel changes from infinity to a finite value. A crude estimate for the magnitude of this spin-mixing contribution to the DOS is

$$
D_{\downarrow}(E) \approx \frac{M_{0}-M_{\mathrm{s}}(T)}{M_{0}+M_{\mathrm{s}}(T)} D_{\uparrow}(E),
$$

where $M_{\mathrm{s}}(T)$ is the spontaneous magnetization and $M_{0}=M_{\mathrm{s}}(0)$. Note that $M_{\mathrm{s}}(T)$ reflects both Bloch-type spin-wave excitations and specific features such as many-sublattice effects.

Spin-resolved photoemission measurements $[3,4]$ that claim to provide evidence of halfmetallic behavior, in fact, do not. Finite-temperature effects leading to the population of spin minority states near $E_{\mathrm{F}}$, just described, would be most significant at wave vectors away from $\bar{\Gamma}$, the surface Brillouin zone center, and may not, therefore, be observed in these measurements at normal emission [3,4], even for stoichiometric surfaces. Similarly, spin minority surface states leading to a loss of half-metallic character, now well described for the half-metallic Heusler alloy NiMnSb $[13,14]$, would not be observed if measurements are only undertaken at the $\bar{\Gamma} k_{\|}=0$ point.

The negligible DOS at the Fermi level in the spin-polarized photoemission spectra of $\mathrm{La}_{2 / 3} \mathrm{Sr}_{1 / 3} \mathrm{MnO}_{3}[3]$ and $\mathrm{CrO}_{2}$ [4] suggests that either the surface is not the correct stoichiometry in those studies (see [15] for $\mathrm{La}_{2 / 3} \mathrm{Sr}_{1 / 3} \mathrm{MnO}_{3}$ and $[16,17]$ for $\mathrm{CrO}_{2}$ ) or that the Fermi level crossings are well away from $\bar{\Gamma}$ (or some combination of both effects). To a small extent, final-state effects in photoemission can also contribute [18]. A band structure like that illustrated schematically in fig. 1b, with a simplified ferromagnetic band, dispersing towards 
a)

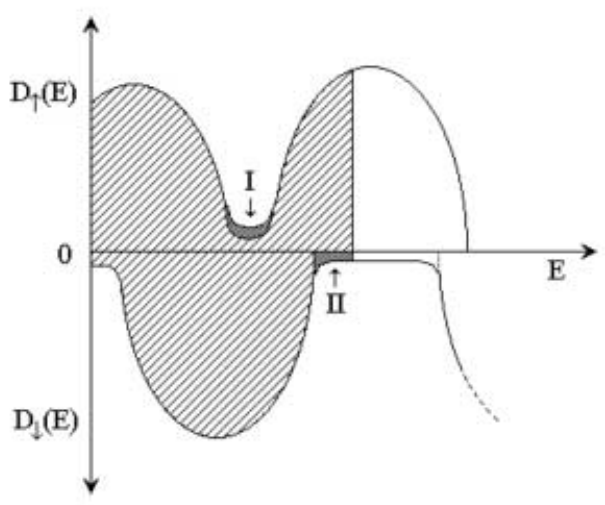

b)

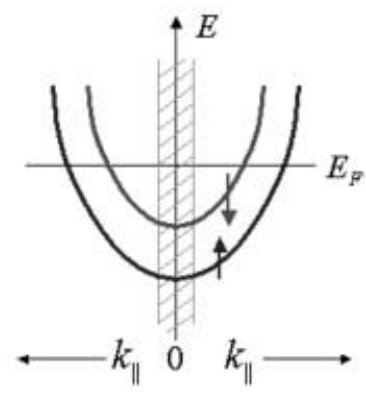

c)

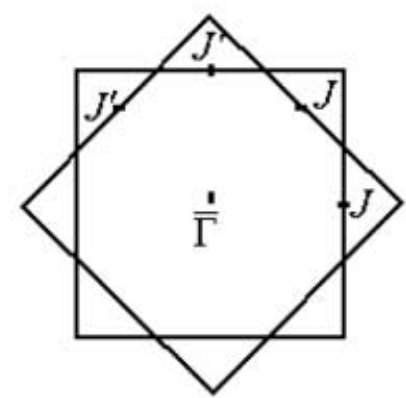

Fig. 1 - Some aspects of the band structure of half-metallic ferromagnets: (a) schematic $\uparrow$ and $\downarrow$ DOS (dark shadowing denotes regions occupied at finite temperature only); (b) a schematic "metallic" ferromagnet band with $\uparrow$ and $\downarrow$ components, indicating that the region sampled at normal emission in photoemission is close to $k_{\|}=0$, and (c) schematic $C_{2 v}$ surface Brillouin zone, applicable to the (001) surfaces of the perovskites and Heusler alloys and illustrating that crystalline texture may rotate the Brillouin zone but leaves the zone center unperturbed.

$E_{\mathrm{F}}$ with increasing wave vectors away from $\bar{\Gamma}$, is not conclusively eliminated in such measurements. The region probed with normal emission in spin-polarized photoemission is the "shaded" region in fig. 1b. Crystalline disorder that might occur $[3,15]$ cannot be used as an argument for "complete" sampling of the Brillouin zone. In such strongly textured thin films, disorder would result in some cylindrical averaging of $k$-points away from $\bar{\Gamma}$ but leave the $\bar{\Gamma}$ point unperturbed, as indicated in fig. 1c.

The onset of "normal" ferromagnetism, with clear evidence of a spin minority population (spin minority scattering) above 80 to $100 \mathrm{~K}$ in the semi-Heusler alloy NiMnSb [2,19] and in $\mathrm{CrO}_{2}$ above 50 to $100 \mathrm{~K}$ [20], is an interesting feature of half-metallic ferromagnets [21]. It is consistent with moment misalignments of the type described by eq. (3). The dramatic decrease in the observed moment of both $\mathrm{Mn}$ and $\mathrm{Ni}$ in $\mathrm{NiMnSb}$, with increasing temperature at about 80 to $100 \mathrm{~K}[2,22]$, suggests that spin alignment is indeed essential to preserve halfmetallic character in NiMnSb. The increase in spin-wave population may also be linked to a "metamagnetic" phase transition between two types of ferromagnetism at about 80 to $100 \mathrm{~K}$.

An increase in spin-wave-induced misalignment is anticipated if the excitation of a static transverse optical mode leads to a lattice distortion that is coupled with the spins in NiMnSb. 
It is already known [23] that a nearly dispersionless transverse optical mode occurs at about $28 \mathrm{meV}$ in NiMnSb. In terms of $3 k_{\mathrm{B}} T$, this corresponds to $103 \mathrm{~K}$ - very close to the temperature at which there is a dramatic loss in Ni and Mn moments in NiMnSb [2]. Spin-wave arguments have already been used to discuss the lower-than-expected Curie temperature of half-metallic materials [24], but an exhausting analysis of the spontaneous magnetization of Heusler alloys and oxides remains a challenge to future research, and full-scale first-principle investigations will be necessary to quantitatively reproduce the observed behavior.

In the case of $\mathrm{La}_{2 / 3} \mathrm{Sr}_{1 / 3} \mathrm{MnO}_{3}$, the appreciable spin minority population at $1.5 \mathrm{~K}$ is probably a band-structure effect [1], involving structural features such as interfaces [13,14], defects [25], and substitutional disorder. An appreciable spin-wave population is unlikely at such low temperatures, even though the spin-wave population is, strictly speaking, nonzero. While a nanocrystalline material with a substantial number of defects will lead to a loss of half-metallic character [25], it will also tend to suppress long-wavelength spin waves.

Unfortunately, indirect proof of half-metallic character from the magnetic moment measured at high fields is likely to be very insensitive to a small spin minority density of states. Even though tunnel junctions include contributions from the spin-dependent Fermi velocity [26], which could effectively enhance the tunnel magneto-resistance $[1,26]$, no tunnel magneto-resistive junctions measurement has provided a strong indication of half-metallic character. The highest tunnel-magnetoresistance values reached have been as high as 450\% [27] to $500 \%$ [28], this only at very low temperatures and still well below the values expected for a half-metal. Transport spin-polarization measurements, using point-contact Andreev reflection, have provided the highest measured polarizations to date $[1,5,29-32]$. In spite of one claim of proof of half-metallic character [5], these measurements also miss the mark of $100 \%$ polarization for the postulated half-metallic systems [1,5,29-32], even at temperatures of $1.6 \mathrm{~K}$ (and lower). So "proof" of half-metallic character must necessarily remain elusive.

In conclusion, our analysis has shown that finite-temperature spin disorder destroys the complete spin polarization characteristic of half-metallic ferromagnets. This mechanism is modified and, in general, enhanced by crystal imperfections, by surface and interface effects, and by specific features of the crystal structure.

$$
* * *
$$

The authors gratefully acknowledge the assistance of HAE-KYUNG JEONG. This research has been supported by NSF (DMR 98-02126), ONR, ARO, AFOSR, and by the Nebraska Research Initiative.

\section{REFERENCES}

[1] Nadgorny B., Mazin I. I., Osofsky M., Soulen R. J., Broussard P., Stroud R. M., Singh D. J., Harris V. G., Arsenov A. and Mukovskit Ya., Phys. Rev. B, 63 (2001) 184433.

[2] Borca C. N., Komesu T., Jeong H., Dowben P. A., Ristoiu D., Hordequin Ch., Nozières J. P., Pierre J., Stadler S. and Idzerda Y. U., Phys. Rev. B, 64 (2001) 052409.

[3] Park J.-H., Vescovo E., Kim H.-J., Kwon C., Ramesh R. and Venkatesan T., Nature, 392 (1998) 794.

[4] Kämper K. P., Schmitt W., Güntherodt G., Gambino R. J. and Ruf R., Phys. Rev. Lett., 59 (1987) 2788.

[5] Ji Y., Strijkers G. J., Yang F. Y., Chien C. L., Byers J. M., Anguelouch A., Xiao G. and Gupta A., Phys. Rev. Lett., 86 (2001) 5585.

[6] Wiesendanger R., Güntherodt H.-J., Güntherodt G., Gambino R. J. and Ruf R., Phys. Rev. Lett., 65 (1990) 247. 
[7] Wei J. Y. T., Yeh N. C. and Vasquez R. P., Phys. Rev. Lett., 79 (1997) 5150.

[8] De Teresa J. M., Barthelemy A., Fert A., Contour J. P., Lyonnet R., Montaigne F., Seneor P. and Vaures A., Phys. Rev. Lett., 82 (1999) 4288.

[9] Irkhin V. Yu. and Katsnel'son M. I., Phys. Usp., 37 (1994) 659.

[10] See, e.g., Sandratskin L. M., Adv. Phys., 47 (1998) 91, and references therein. An illustrative example of the related effect of local spin disorder on the DOS of "simple" ferromagnets has been given by Sandratskit L. M. and KüBler J., fig. 6 in Magnetism and Electronic Correlations in Local Moment Systems, edited by Donath M., Dowben P. A. and Nolting W. (World Scientific, Singapore) 1998, p. 271.

[11] Lorenz R., Hafner J., Jaswal S. S. and Sellmyer D. J., Phys. Rev. Lett., 74 (1995) 3688.

[12] Skomski R. and Coey J. M. D., Permanent Magnetism (Institute of Physics, Bristol) 1999.

[13] Jenkins S. J. and King D. A., Surf. Sci. Lett., 501 (2002) L185.

[14] De Wijs G. A. and De Groot R. A., Phys. Rev. B, 64 (2001) 020402.

[15] Dulli H., Plummer E. W., Dowben P. A., Choi J. and Liou S.-H., Appl. Phys. Lett., 77 (2000) 570.

[16] Dai J., Tang J., Xu H., Spinu L., Wang W., Wang K.-Y., Kumbhar A., Li M. and Diebold U., Appl. Phys. Lett., 77 (2000) 2840.

[17] Cheng R.-H., Xu B., Borca C. N., Sokolov A., Yang C.-S., Yuan L., Liou S.-H., Doudin B. and Dowben P. A., Appl. Phys. Lett., 79 (2001) 3122.

[18] Joynt R., Science, 284 (1999) 777.

[19] Hordequin Ch., Ristoiu D., Ranno L. and Pierre J., Eur. Phys. J. B, 16 (2000) 287.

[20] Watts S. M., Wirth S., von Molnár S., Barry A. and Coey J. M. D., Phys. Rev. B, 61 (2000) 9621.

[21] Coey J. M. D., to be published in J. Appl. Phys.

[22] Hordequin Ch., Lelievre-Berna E. and Pierre J., Physica B, 234-236 (1997) 602.

[23] Borca C. N., Ristoiu D., Komesu T., Jeong H., Hordequin Ch., Pierre J., Nozières J. P. and Dowben P. A., Appl. Phys. Lett., 77 (2000) 88.

[24] Liu S. H., J. Magn. \&3 Magn. Mater., 212 (2000) 259.

[25] Orgassa D., Fujiwara H., Schulthess T. C. and Butler W. H., Phys. Rev. B, 60 (1999) 13237.

[26] Mazin I. I., Phys. Rev. Lett., 83 (1999) 1427.

[27] Viret M., Nassar J., Drouet M., Contour J. P., Fermon C. and Fert A., J. Magn. 63 Magn. Mater., 198-199 (1999) 1.

[28] Versluijs J. J., Bari M. A. and Coey J. M. D., Phys. Rev. Lett., 87 (2001) 6601.

[29] Soulen R. J., Byers J. M., Osofsky M. S., Nadgorny B., Ambrose T., Cheng S. F., Broussard P. R., Tanaka C. T., Nowak J., Moodera J. S., Barry A. and Coey J. M. D., Science, 282 (1998) 85.

[30] Osofsky M. S., Soulen R. J., Nadgorny B. E., Trotter G., Broussard P. R. and DeSisto W. J., Mat. Science Eng. B, 84 (2001) 49.

[31] Osofsky M. S., Nadgorny B., Soulen R. J., Trotter G., Broussard P., Desisto W., Laprade G., Mukovskit Y. M. and Arsenov A., Physica C, 341 (2000) 1527.

[32] Desisto W. J., Broussard P. R., Ambrose T. F., Nadgorny B. E. and Osofsky M. S., Appl. Phys. Lett., 76 (2000) 3789. 